\title{
Tracer: A general-purpose software library for logging events in computerized experiments
}

\author{
Olaf Lahl and Reinhard Pietrowsky \\ University of Düsseldorf, Düsseldorf, Germany
}

\begin{abstract}
In certain areas of the behavioral sciences, such as cognitive and perceptual psychology, researchers may choose to have their experiments partially or completely driven by software programs, which instruct and guide subjects through the sequence of tasks. Despite distinct advantages of unattended trial execution, on frequent occasions, experimenters may desire to keep track of the progress or to be notified of certain events, such as when the subject has completed a task. The Tracer software library presented here is a lightweight Windows programming interface that provides experimenters the ability to trace events and status notifications on one or more remote computers and log files. With only a few lines of additional code or script code, researchers can monitor the real-time progress of one or more unattended experiments running on remote computers of the local area network or the Internet. This article describes the functionality and usage of the Tracer library. The Tracer binaries, include files, sample code, and documentation files may be downloaded from the Psychonomic Society Archive of Norms, Stimuli, and Data at www.psychonomic.org/archive.
\end{abstract}

Today, the widespread use of information technology is a matter of course in most areas of behavioral research. Classical methods of experimentation, such as reading stimuli aloud; presenting items on memory drums, paper cards, or tachistoscopes; and the tape recording of oral output have largely been replaced by software applications with an ever-increasing level of sophistication, ease of use, and accuracy. Depending on the trade-off between flexibility and total cost of implementation, researchers can choose from a wide range of technologies for implementing their experimental design with handwritten assembly and $\mathrm{C} / \mathrm{C}++$ code on one end of the scale and high-level scripting tools on the other (see Schneider, Bolger, Eschman, Neff, \& Zuccolotto, 2005, for an overview of common experiment-generation software tools).

Complete or partial computerization of the experimental protocol offers distinct advantages over classic routines in terms of timing accuracy, ease of data collection, and strict standardization of treatments and instructions given to subjects. Regarding the latter, elimination of the experimenter from the experimental situation is certainly the best method for avoiding effects of the experimenter's expectations and attributes on subjects' behavior (see Rosenthal, 1976, for an extensive review of this line of research). Nevertheless, although many experimenters would readily agree to be removed from the experimental session, they may still desire to have some level of monitoring control over the course of the preprogrammed events through which the subjects are passing during the experiment.

With the Tracer software library presented here, researchers can take full advantage of completely auto- mated experimentation while still having real-time access to the sequence of experimental events. The library is programmed for Microsoft Windows platforms and consists of a small set of functions with a straightforward and common usage. With a minimum of overhead, experimenters can call these functions from within their source or script code in order to log any event of interest during the course of program execution to one or more remote computers and log files. The Tracer library differs from existing logging applications (Alexander, Cockburn, \& Lobb, 2008; Trewin, 1998) that can operate on unmodified software applications but are restricted to low-level events, such as single keystrokes, mouse buttonpresses, scrolling events, and so forth, to local files. Subsequent offline analysis of such events is needed in tests of software usability and user interface design and may also be adequate for simple reaction time tasks. However, many other types of experiments require a much more verbose and elaborative remote tracing of events in real time. By calling Tracer functions, experimenters can accomplish this task for user-defined events of any complexity that may seem appropriate within the context of a given experiment.

To our knowledge, the idea of logging information about the sequence of experimental events to a remote monitor program has so far been implemented only by DMDX, an experiment-generation tool primarily targeted at language-processing experiments (Forster \& Forster, 2003). DMDX writes the currently executing line of source code to a remote computer as the experiment is running. The Tracer library, on the other hand, provides more flex-

O.Lahl, olaf.lahl@uni-duesseldorf.de 
ible, general-purpose event logging for different kinds of experiments, tools, and programming languages.

\section{Description of the Software}

The Tracer software consists of two parts - a source (server) and a sink (client) part-with a unidirectional flow of information from server to client, but not vice versa (Figure 1). The dynamic link library Tracer.dll is the server component that Windows developers can use from within their source or script code running the experiment. It dispatches trace events to log files, as well as to remote computers running the client application TraceMon.exe. The trace monitor acts like a live ticker, reporting line by line the events from one or more trace sources. Trace sources report to remote trace sinks via the user datagram Internet protocol. Therefore, communication between trace sinks and sources is not restricted to local area networks, but covers the area of the Internet.

The server component exposes its functionality via a set of exported $\mathrm{C}$ functions and via an automation-compatible common object model (COM) interface, ITracer. ${ }^{1}$ Whereas the $\mathrm{C}$ calls are intended for applications written in common third-generation languages, such as $\mathrm{C}, \mathrm{C}++$, or Pascal, the COM interface makes the Tracer implementation available to higher level macro and scripting languages, such as Visual Basic for Applications, VBScript, JScript, and any other scripting language that supports object linking and embedding (OLE) automation (see Rogerson, 1997, for details on COM and OLE automation). Most important, the automation interface makes the Tracer library available to E-Basic (a Visual Basic dialect) scripts developed with E-Prime (Schneider, Eschman, \& Zuccolotto,

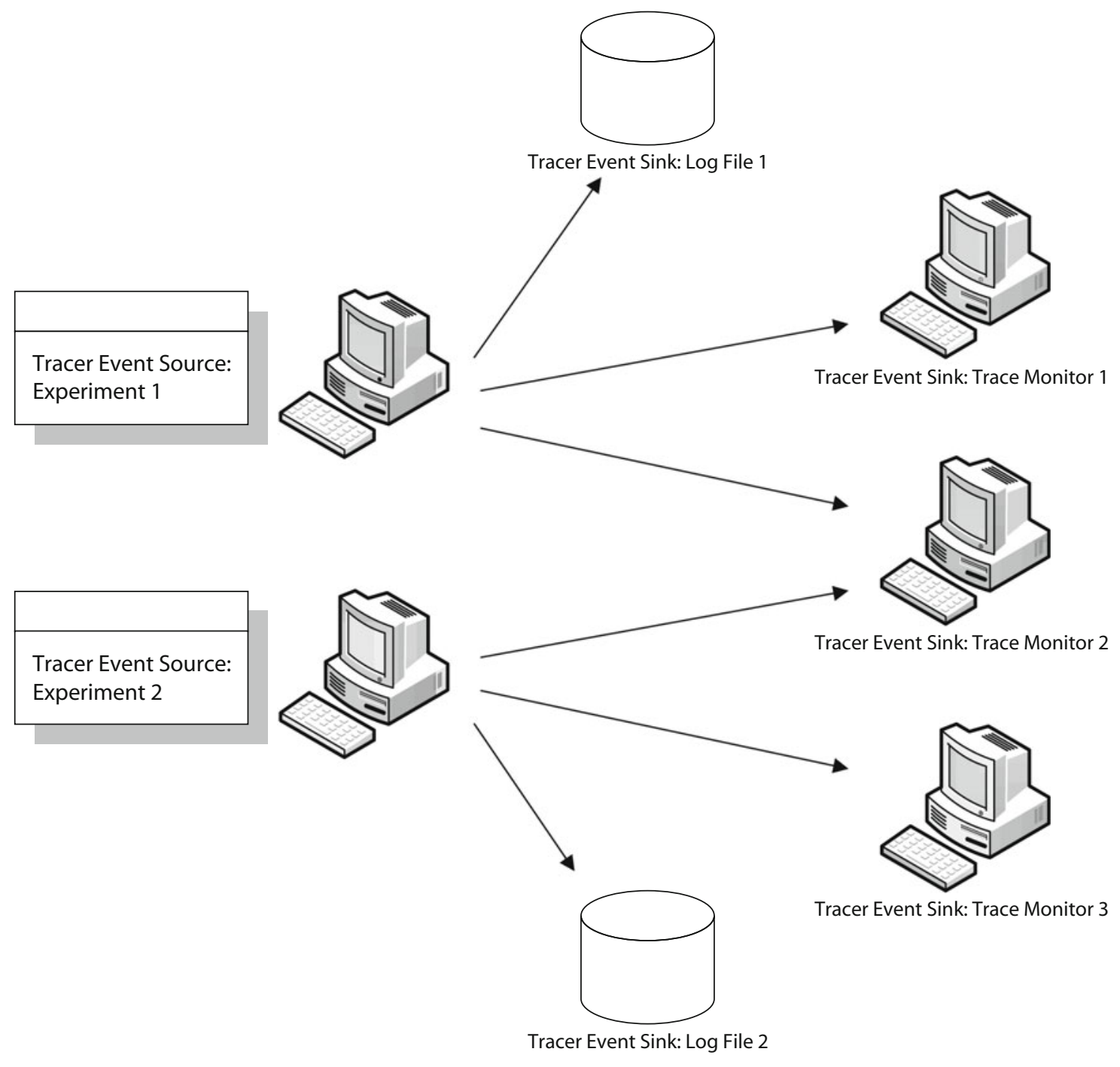

Figure 1. Information flow between Tracer event sources and event sinks. A Tracer event source is a running executable or script utilizing Tracer calls exported by Tracer. dll. A Tracer event sink is a log file or a remote computer running the Trace Monitor (TraceMon.exe). An event source can target multiple sinks, and a sink can receive input from multiple event sources. 
2002), probably the most popular experiment-generation tool.

\section{Using the Tracer Library}

Using the Tracer Library involves the following steps:

1. Initialize the Tracer Library by calling TrcInit.

2. Register one or more trace targets by calling TrcRegisterMachine for remote computers and TrcRegisterLogFile for $\log$ files.

3. Subscribe to one or more keyboard or mouse events by calling TrcSubscribeKeyStroke or TrcSubscribeMouseClick. After successful subscription, these functions will automatically trace the message string you pass whenever the specified key is hit or mouse button is pressed.

4. Trace events by calling TrcTrace for every event of interest, passing the message string and arguments to be sent to all trace targets that were registered in Step 2.

5. To remove a trace target from the trace chain, call TrcUnregisterMachine and TrcUnregisterLogFile, in that order.

6. To unsubscribe from an event, call TrcUnsubscribeKeyStroke and TrcUnsubscribeMouseClick, in that order.

7. Clean up the Tracer library by calling TrcExit.

Appendix A provides a code snippet that illustrates a typical use of the Tracer library by $\mathrm{C} / \mathrm{C}++$ clients. Appendix $\mathrm{B}$ shows a functionally equivalent code sample as it would be implemented by a Visual Basic automation client. A complete library reference is available at www .psychonomic.org/archive. The Tracer output in the Trace Monitor, derived from these code snippets, is reproduced in Figure 2.

The following implementation details should be kept in mind when planning to deploy the Tracer library:

1. TrcInit must be the first Tracer call.

2. TrcExit should be called when the Tracer library is no longer needed, in order to clean up all resources used.

3. Most Tracer calls return a Boolean value of nonzero after successful execution and zero in case of failure. For any of these functions, you may call TrcGetLastError or TrcGetLastErrorString in order to obtain either the Win 32 error code or the string of the calling thread's last error.
4. The core function TrcTrace accepts a format string as the first argument and a variable list of arguments thereafter. The syntax is equivalent to that of the $\mathrm{C}$ standard library function printf.

5. The Tracer library automatically time stamps each tracing output. You do not have to provide your own code for generating time stamps.

6. As an alternative to Steps 5 and 6 above, you may call TrcUnregisterAllTraceTargets in order to remove all trace targets and event subscriptions at once.

7. All Tracer functions are thread safe (threading model "free" for the COM interface), meaning that they can be called simultaneously from multiple threads, without the risk of race conditions.

\section{Software Requirements and Availability}

The Tracer software development kit, including the Tracer binaries, include files, samples, and documentation files - as well as the Trace Monitor application — can be downloaded at no cost at ftp://ftp.uni-duesseldorf.de/pub/ psycho/lahl/Tracer. Trace Monitor is a Windows .NET application that runs on any platform that supports Version 2.0 or higher of the Microsoft .NET framework. The Tracer server component Tracer.dll requires Version 5.1 (which corresponds to Windows XP) or a higher version of the Microsoft Windows operating system. The compiled code comes in two versions that offer native support for 32bit and 64-bit platforms. Authors who use the Tracer library for a published work are expected to cite this reference.

\section{DISCUSSION}

With the Tracer library presented in this article, we provide a lightweight application programming interface for logging user-defined events to remote computers and log files. With remote computers and file tracing, it becomes possible to (1) routinely monitor the execution of otherwise unattended computerized experiments in real time, (2) provide precise ex post facto evidence about the course of experimental events, and (3) exchange online status information in multiuser programs (e.g., judgment and decision-making programs, computerized microworlds). In our own sleep laboratory, we routinely use the Tracer library to keep track of our subjects' behavior during testing and to alert the experimenter in case of unexpected events

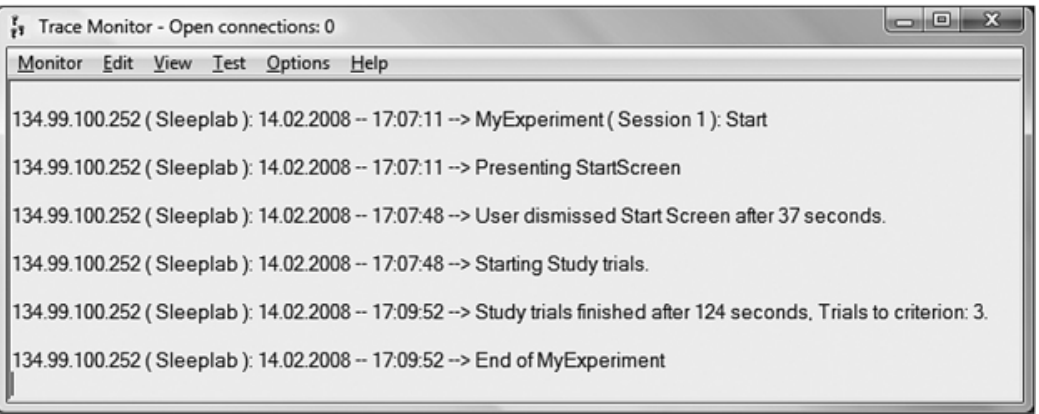

Figure 2. Tracer output from Appendixes A and B in a Trace Monitor window. 


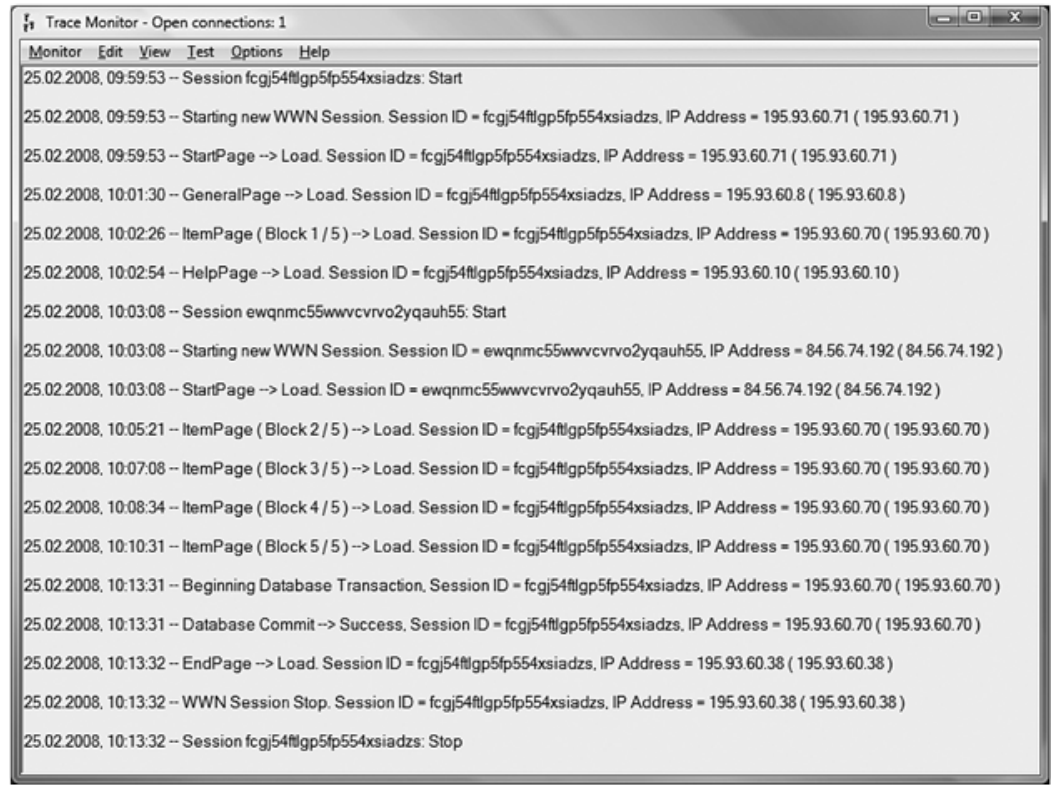

Figure 3. Snapshot of a Tracer output in a Trace Monitor window from one of our own Web experiments.

(e.g., nonresponding subjects). We also make extensive use of tracing in our Web-based studies (Figure 3), which gives us status information instantly and with much more detail than the offline log files of the Web server. ${ }^{2}$

\section{AUTHOR NOTE}

Correspondence concerning this article should be addressed to O. Lahl, Institute of Experimental Psychology, University of Düsseldorf, Universitätsstr. 1, D-40225 Düsseldorf, Germany (e-mail: olaf.lahl@, uni-duesseldorf.de).

\section{REFERENCES}

Alexander, J., Cockburn, A., \& LobB, R. (2008). AppMonitor: A tool for recording user actions in unmodified Windows applications. Behavior Research Methods, 40, 413-421.

Forster, K. I., \& Forster, J. C. (2003). DMDX: A Windows display program with millisecond accuracy. Behavior Research Methods, Instruments, \& Computers, 35, 116-124.

Rogerson, D. E. (1997). Inside COM: Microsoft's component object model. Redmond, WA: Microsoft Press.

Rosenthal, R. (1976). Experimenter effects in behavioral research (Rev. ed.). New York: Irvington.

Schneider, W., Bolger, D. J., Eschman, A., NefF, C., \& Zuccolotto, A. P. (2005). Psychology experiment authoring kit (PEAK): Formal usability testing of an easy-to-use method for creating computerized experiments. Behavior Research Methods, 37, 312-323.
Schneider, W., Eschman, A., \& Zuccolotto, A. [P.] (2002). E-Prime user's guide. Pittsburgh: Psychology Software Tools.

TRewIN, S. (1998). InputLogger: General-purpose logging of keyboard and mouse events on an Apple Macintosh. Behavior Research Methods, Instruments, \& Computers, 30, 327-331.

\section{NOTES}

1. To be more precise, ITracer is a dual interface inherited from IDispatch.

2. Incidentally, we hereby detected a recent denial-of-service attack on our Web server at a very early stage.

\section{ARCHIVED MATERIALS}

The following materials associated with this article may be accessed through the Psychonomic Society's Norms, Stimuli, and Data Archive, www.psychonomic.org/archive.

To access these materials, search the archive for this article using the journal name (Behavior Research Methods), the first author's name (Lahl), and the publication year (2008).

FILE: Lahl-BRM-2008.zip

DESCRIPTION: The compressed archive file contains two files:

/Win32/Tracer.msi, containing a setup file for Windows 32-bit platforms (Windows XP or higher)

Win64/Tracer.msi, containing a setup file for Windows 64-bit platforms (Windows XP 64-bit or higher)

AUTHOR’s E-MAIL ADDRESS: olaf.lahl@uni-duesseldorf.de. 


\section{Code Example for $\mathrm{C} / \mathrm{C}++$ Clients on How to Use the Tracer Software Library}

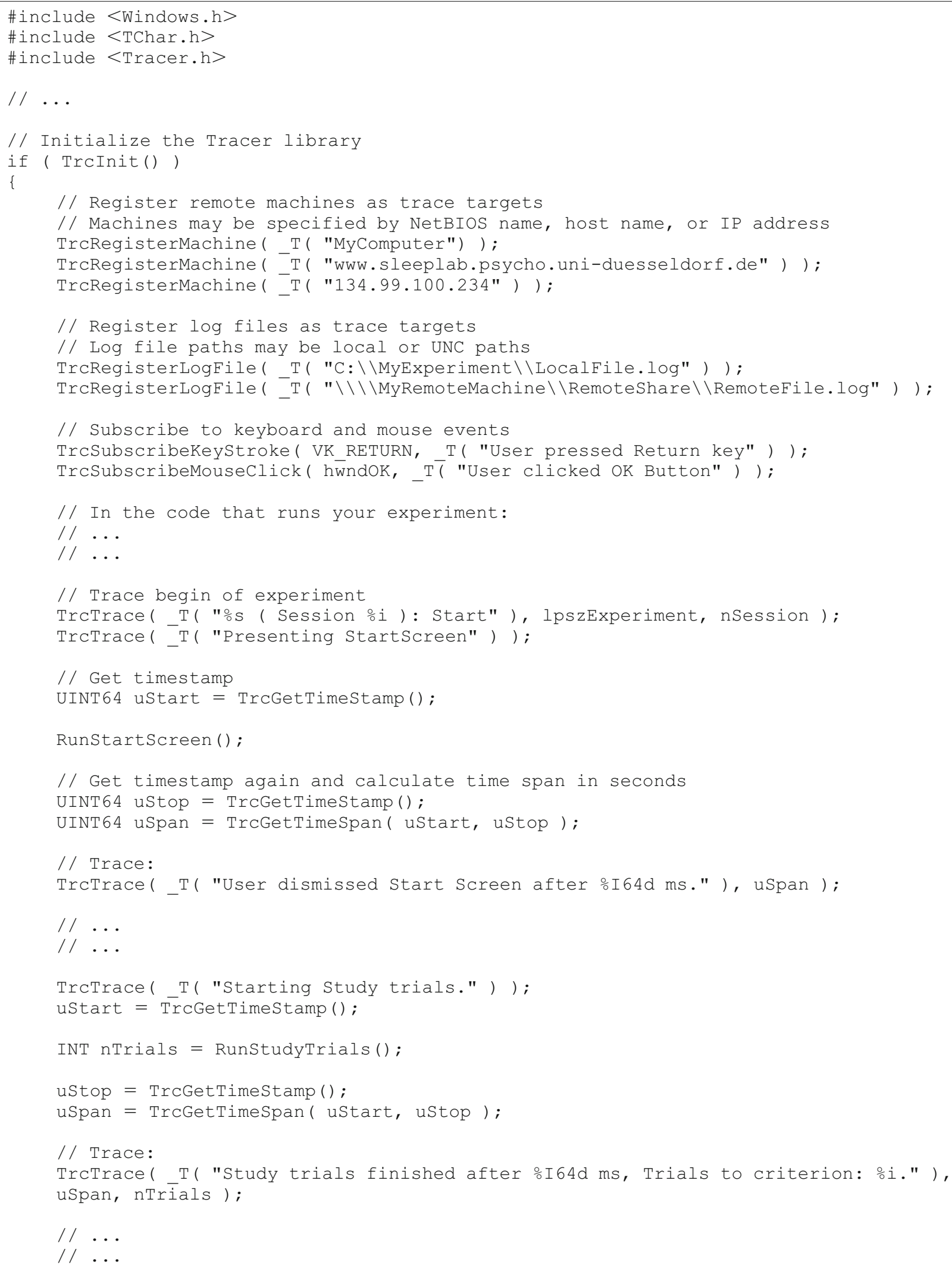


APPENDIX A (Continued)

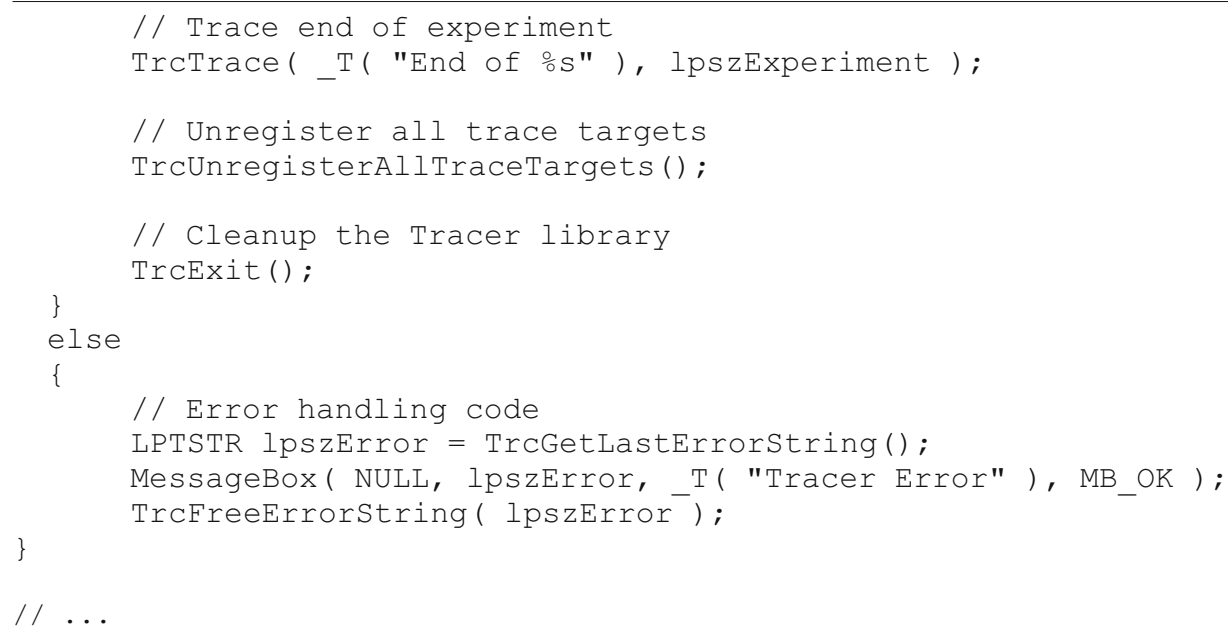

\section{APPENDIX B \\ Code Example for Visual Basic Automation Clients on How to Use the Tracer Software Library, Functionally Equivalent to That of Appendix A}

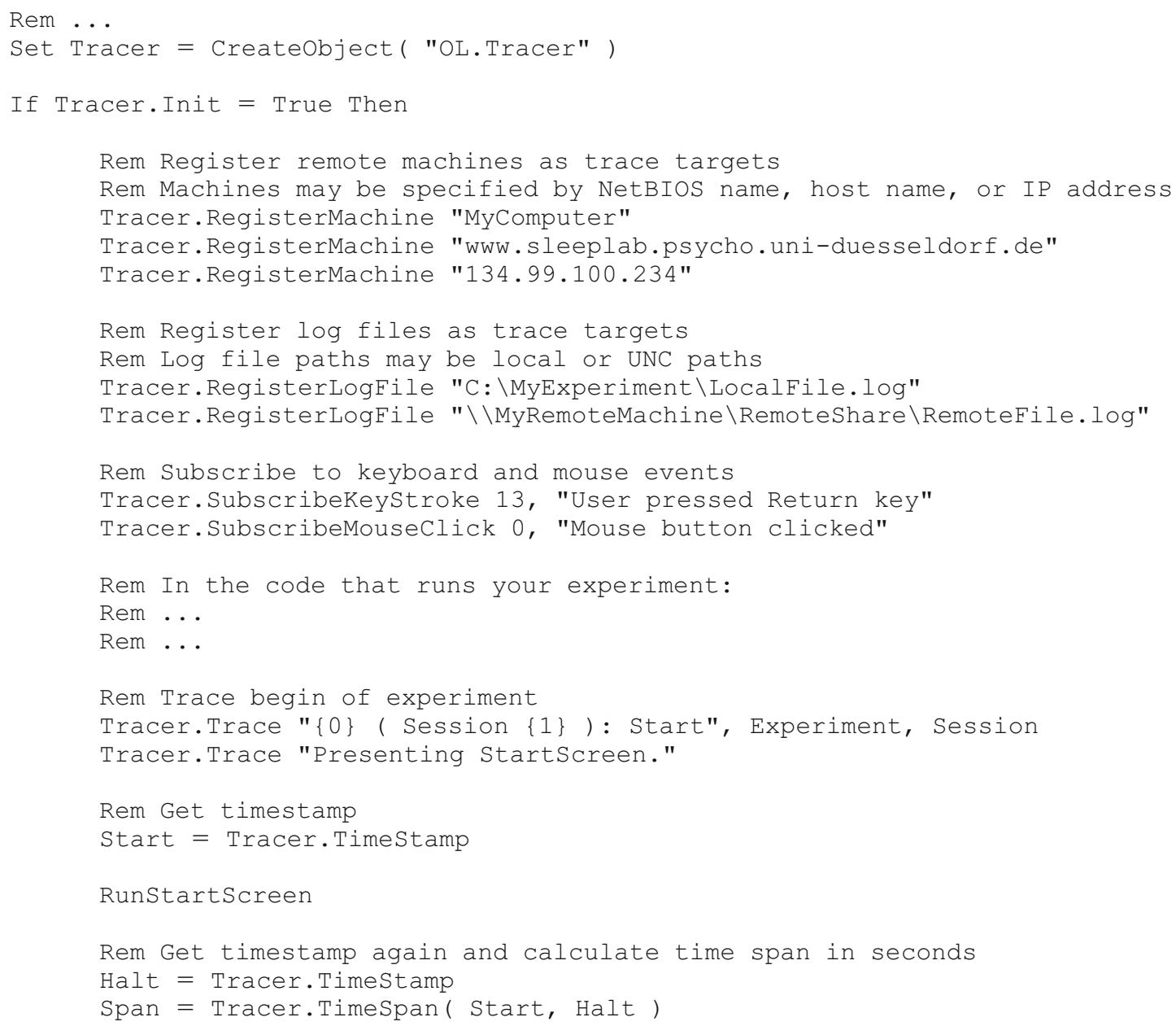




\section{APPENDIX B (Continued)}

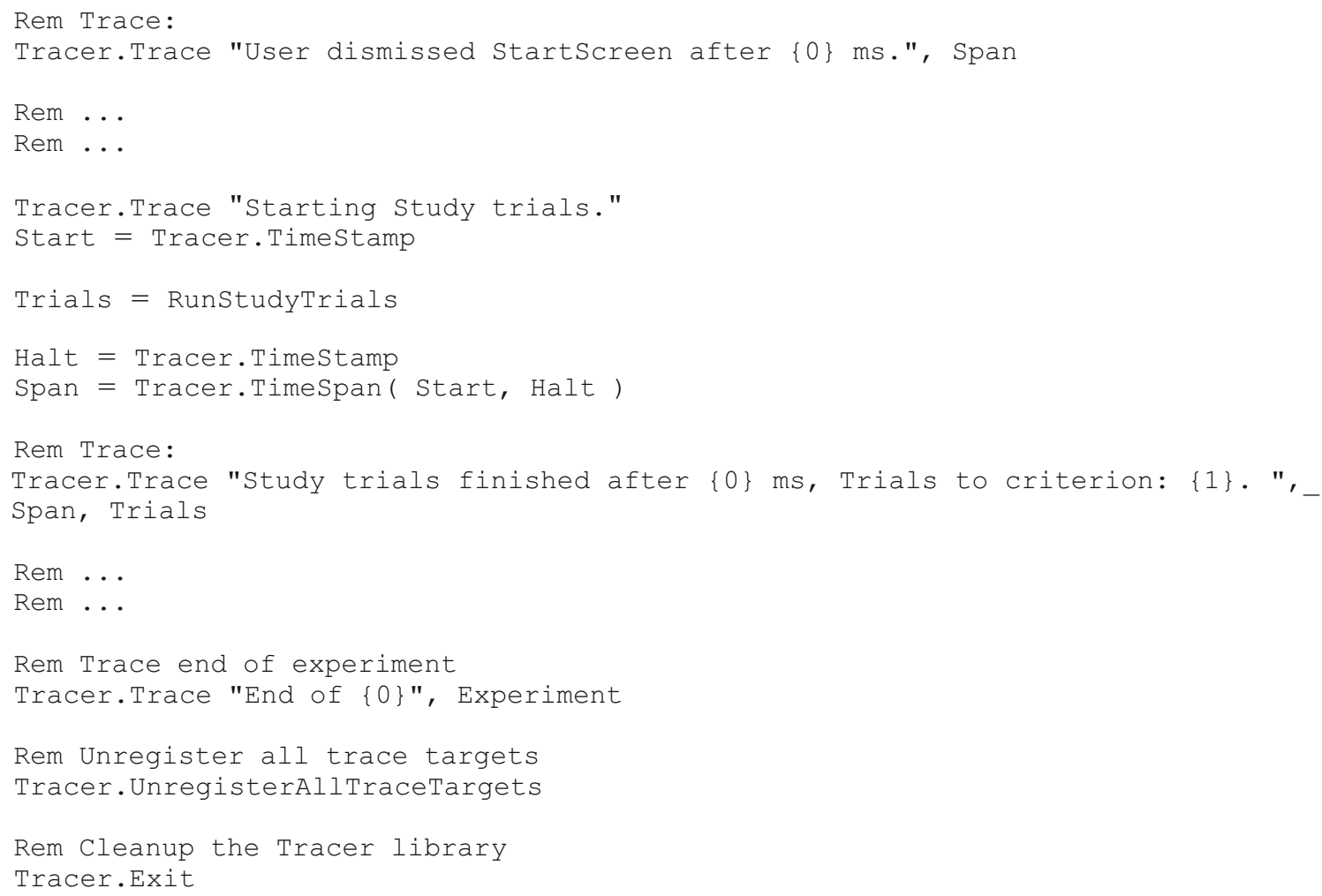

